\title{
The Co-Discovery of Conservation Laws and Particle Families
}

\author{
Oliver Schulte ${ }^{1, *}$ \\ Department of Philosophy and School of Computing Science, \\ Simon Fraser University, \\ Burnaby, B.C., V5A 1S6, Canada
}

\begin{abstract}
This paper presents an epistemological analysis of the search for new conservation laws in particle physics. Discovering conservation laws has posed various challenges concerning the underdetermination of theory by evidence, to which physicists have found various responses. These responses include an appeal to a plenitude principle, a maxim for inductive inference, looking for a parsimonious system of generalizations, and unifying particle ontology and particle dynamics. The connection between conservation laws and ontological categories is a major theme in my analysis: While there are infinitely many conservation law theories that are empirically equivalent to the laws physicists adopted for the fundamental Standard Model of particle physics, I show that the standard family laws are the only ones that determine and are determined by the simplest division of particles into families.
\end{abstract}

Key words: Conservation Laws, Underdetermination, Plenitude, Simplicity, Natural Kinds, Induction

\section{Introduction: Conservation Laws and Underdetermination}

The underdetermination of belief by evidence is a central topic in epistemology, as it is the point of departure for skeptical arguments against the possibility of knowledge. One aim of the philosophy of science is to study underdetermination as it arises in scientific practice. Such case studies refine our understanding

* Corresponding author.

Email address: oschulte@cs.sfu.ca (Oliver Schulte ).

1 This research was supported by a research grants from the Natural Sciences and Engineering Research Council of Canada and the Social Sciences and Humanities Research Council of Canada.

Preprint submitted to Elsevier Science 8 January 2008 
by revealing different kinds of underdetermination. In addition, the epistemological problems that scientists face are rich and complex, and since scientists often confront them with success, the practice of science promises to teach us much about effective strategies for responding to the challenge of underdetermination. Moreover, a systematic philosophical analysis of the relationship between theory and evidence in a scientific domain can clarify the structure and the strength of the logic we find in practice.

In this paper I consider how physicists have resolved various problems of underdetermination that arise in the search for a theory of reactions among elementary particles. One type of underdetermination is global underdetermination, which arises when even complete knowledge of all observational facts does not determine the answer to the scientific question under investigation. (My use of the terms 'global' and 'local' underdetermination follows Kelly's thorough discussion [16, Ch.2].) Global underdetermination is a concern in particle physics in at least two ways. First, a possible reaction among elementary particles may never materialize, in which case it will never be observed, and the observational facts may not determine whether the reaction is possible or not. Second, for a given set of (additive) conservation laws there are infinitely many different laws that are consistent with exactly the same processes among elementary particles. Thus even complete knowledge of all reactions ever to occur does not determine a unique set of laws without further considerations.

Local underdetermination arises when finite data samples do not determine the answer to a scientific question, even if complete knowledge of the infinitely many observational facts would. Local underdetermination is closely related to the classic problem of induction. An example of local underdetermination in particle physics is the issue of whether a reaction that has not been observed yet will be observed in a future experiment. Since conservation principles entail assertions about what reactions can and cannot be observed, the local underdetermination of possible reactions means that finite data may not determine which conservation principles make correct predictions.

The final type of underdetermination that I consider in this paper is the underdetermination of particle ontology by empirical phenomena. One of the goals of particle physics is to find a taxonomy for the particle zoo that relates properties of particles to empirical phenomena. The main result of this paper is that data about observed reactions among elementary particles determine an essentially unique simplest combination of particle classes and conservation laws. This finding supports and illustrates David Lewis' observation that "laws and natural properties get discovered together" [20, p.368].

This paper is not a historical account of the development of particle conservation laws from the 60 s through the 80 s (for the history of the subject, see, e.g., $[8,22])$. Instead I focus on some of the epistemological issues that arose in 
the development of conservation laws. With regard to historical and current practice, my study aims to be realistic, but within a limited scope. It is realistic in that I consider laws of a form found in actual particle theory - additive conservation laws - and I analyze actual reaction data from particle accelerators. It is limited in various respects, such as: (1) I leave aside other types of conservation laws (e.g., discrete spacetime symmetries [41,3,42]). (2) I consider the formulation of conservation laws as a topic on its own, rather than in combination with the development of quark theory and the fundamental Standard Model of particle physics. (3) I do not analyze attempts to derive inductively inferred conservation laws from more fundamental principles - what Earman calls the search for "second-order laws" [5]. (For instance, I do not discuss explaining the conservation of Baryon number via quark confinement and quantum chromodynamics. For a textbook discussion of this part of theoretical physics, see [40]. Nor does this paper address string theory.) Thus the view of additive conservation laws in this paper is closer to the phenomenological approach of the 1950s and 1960s than to current theories about conservation laws. As far as the history of particle theory is concerned, my analysis is best viewed as pertaining to an early historical period of particle research.

Despite these limitations, my discussion includes enough aspects of particle theory to raise and illuminate many philosophical questions about the underdetermination of theory and ontology by evidence. Also, the fact that the epistemological analysis matches the physicists' inferences, as I show below, indicates that my discussion captures much of the logic that guided the discovery of selection rules in particle research.

The paper is organized as follows. In section 2 I outline the project of discovering new conservation laws in particle physics, and the principles that have guided this project as formulated by prominent practioners such as Nobel Laureates Feynman and Cooper. Section 3 analyzes the logical structure of additive conservation laws - what empirical predictions can they express, and under what circumstances do different sets of laws make the same predictions? Following the general principles of scientific inference examined in Section 2, I formulate the maximally strict inference rule: posit conservation principles that explain the nonoccurrence of as many unobserved particle reactions as possible. Section 4 examines the status of the maximally strict inference rule. This rule can be justified in terms of a general inductive epistemology whose precept is that good inference rules serve the aims of inquiry (in the present analysis, the aims of true and stable belief). The main finding of this section is that given the particle reaction data that are currently available, physicists adopted conservation laws that follow the maximally strict inference rule. As there are infinitely many alternative law sets that are empirically equivalent to those physicists adopted, the question arises what is special about the standard laws. Section 6 answers this question by showing that the standard laws correspond to a simple division of elementary particles into disjoint families, 
and that they are essentially the only laws to do so. Section 7 develops the idea that in the particle domain, parsimony is a guide to ontological simplicity: with the aid of a computer program we show that maximizing a natural measure of the parsimony of a set of laws leads to the rediscovery of the standard particle families and the corresponding conservation laws. The enterprise of particle physics raises some of the great themes of the philosophy of science: natural laws, natural kinds, and simplicity. In the final section I comment on David Lewis' prominent views on these topics in the light of what my case study shows about conservation laws in particle physics.

\section{Conservation Laws and Underdetermination in Particle Physics}

I use the term "global underdetermination" to refer to a situation with two possible worlds in which our experience is exactly the same [16]. This scenario is familiar in philosophy. For instance, Descartes' Meditations described two worlds in which our experience is the same, one in which an evil demon produces our illusions, and another in which our perceptions reflect reality. In particle physics, the possibility of global underdetermination arises in a more pedestrian manner. Consider a possible physical process such as $p \rightarrow \pi^{0}+e^{+}$, the decay of a proton into a pion and a positron. Is it possible that the laws of physics permit this process, yet we never observe it? If yes, then global underdetermination arises: there are two physically possible worlds, one in which $p \rightarrow \pi^{0}+e^{+}$is a possible process, another in which the process is forbidden, yet our total evidence in each may be the same.

This possibility may seem unlikely, and indeed particle physicists have explicitly ruled it out.

There is an unwritten precept in modern physics, often facetiously referred to as Gell-Mann's totalitarian principle, which states that 'anything which is not prohibited is compulsory'. Guided by this sort of argument we have made a number of remarkable discoveries from neutrinos to radio galaxies. $[1]$

So the scenario I sketched above cannot happen: If $p \rightarrow \pi^{0}+e^{+}$is not forbidden, then by Gell-Mann's Totalitarian principle, the process must eventually occur. ${ }^{2}$ Kenneth Ford states a succinct version of the Totalitarian principle that emphasizes the role of conservation laws: "everything that can happen

$\overline{2}$ Surely Gell-Mann's principle is meant with the caveat that it applies only provided the necessary initial conditions for a process to take place are created sufficiently often. For example, current particle theory holds that the collision of two protons may result in any number of pions if the two protons meet with sufficient energy. In 
without violating a conservation law does happen" [8, p.82], Ford's emphasis. The next section elaborates on the importance of conservation laws in the search for the laws of particle dynamics.

If the concerns of global underdetermination are met, the total findings of an unbounded course of inquiry would settle all questions about what particle reactions are possible. However, at a given stage of inquiry, physicists will have explored but a finite amount of phenomena and gathered but a finite number of observations. Thus the challenge remains of how to generalize from the evidence available in the short run. As there are an infinite number of particle theories logically consistent with a finite amount of data, we face a problem of local underdetermination.

As Richard Kane astutely observes, the Totalitarian Principle not only defuses global underdetermination, but also carries implications for the direction of research and theory in particle physics [14]:

What is interesting is that, in committing themselves to plenitude in this restricted form, modern physicists are committing themselves to the principle that what never occurs must have a sufficient reason or explanation for its never occurring.

Thus the Totalitarian Principle implies an explanatory strategy: to focus on reactions that do not occur as the primary explananda. If we recall Ford's conservation law version of the Totalitarian Principle, the strategy is to find quantities that are conserved in reactions known to be possible, but not conserved in problematic reactions that fail to be observed. Nobel Laureate Leon Cooper testifies to the importance of conservation principles, in particular selection rules (a type of conservation law that I will discuss in detail presently) $[2, \mathrm{p} .458]$.

In the analysis of events among these new particles, where the forces are unknown and the dynamical analysis, if they were known, is almost impossibly difficult, one has tried by observing what does not happen to find selection rules, quantum numbers, and thus the symmetries of the interactions that are relevant.

Richard Feynman also emphasizes the role of selection rules (as represented in tables, see Table 1 below) in his famous artice on The Great Conservation

symbols, the process $p+p \rightarrow p+p+\overbrace{\pi^{0}+\pi^{0}+. .+\pi^{0}}^{n}$ is possible for any $n$ provided the two colliding protons have enough energy. But there is no reason to think that either nature left to itself or physicists will invest enough energy in proton-proton collisions to produce, say, one thousand pions. I owe this observation to James T. Brown. 
Laws.

The reason why we make these tables [of conserved quantities] is that we are trying to guess at the laws of nuclear interaction, and this is one of the quick ways of guessing at nature [7, p.67].

These sources make clear that the emphasis on conservation principles has been a great help to physicists in their search for a theory of partice reactions. Much of this paper is concerned with working out exactly how. The assumption that conservation principles are adequate for describing particle dynamics rules out many logically possible particle worlds and thus amounts to a substantive empirical background assumption that limits skeptical possibilities. To see why that is so, we need to study the logical structure and predictive import of conservation principles in some detail.

\section{The Logic of Additive Conservation Laws}

This section introduces additive conservation principles and discusses their status in the fundamental Standard Model of Particle Physics and recent extensions of the Standard Model. An analysis follows of how the focus on additive conservation principles can resolve local underdetermination.

\subsection{Conservation Laws in Particle Physics}

A particle is an object that obeys the rules of quantum mechanics for a point with well-defined mass and charge [23, Ch.1.1]. Physicists refer to particles that are neither atoms nor nuclei as 'elementary' particles. ${ }^{3}$ Several kinds of conservation principles in particle physics describe what reactions among elementary particles are possible: General conservation principles such as conservation of energy, momentum and electric charge, discrete space-time symmetries such as parity and CPT, and so-called numeric or additive conservation principles $[41,42]$. My analysis considers the last kind of conservation principle, also known as a selection rule [23, p.36]. A selection rule introduces a quantity and assigns a value for that quantity to each known elementary particle. Table 1 lists 5 such quantities, namely electric charge, baryon number, electron number, muon number and tau number; it specifies what value each of the 22 listed common particles has for a given quantity [41]. These quantities are the same for each kind of particle in all reactions (unlike, say, momentum). From now

3 except for the proton, which is considered an elementary particle although it is the nucleus of the hydrogen atom. 


\begin{tabular}{|c|c|c|c|c|c|c|}
\hline & Particle & Charge (C) & Baryon\# (B) & Tau\# (T) & Electron\# (E) & Muon\# (M) \\
\hline 1 & $\Sigma^{-}$ & -1 & 1 & 0 & 0 & 0 \\
\hline 2 & $\bar{\Sigma}^{+}$ & 1 & -1 & 0 & 0 & 0 \\
\hline 3 & $n$ & 0 & 1 & 0 & 0 & 0 \\
\hline 4 & $\bar{n}$ & 0 & -1 & 0 & 0 & 0 \\
\hline 5 & $p$ & 1 & 1 & 0 & 0 & 0 \\
\hline 6 & $\bar{p}$ & -1 & -1 & 0 & 0 & 0 \\
\hline 7 & $\pi^{+}$ & 1 & 0 & 0 & 0 & 0 \\
\hline 8 & $\pi^{-}$ & -1 & 0 & 0 & 0 & 0 \\
\hline 9 & $\pi^{0}$ & 0 & 0 & 0 & 0 & 0 \\
\hline 10 & $\gamma$ & 0 & 0 & 0 & 0 & 0 \\
\hline 11 & $\tau^{-}$ & -1 & 0 & 1 & 0 & 0 \\
\hline 12 & $\tau^{+}$ & 1 & 0 & -1 & 0 & 0 \\
\hline 13 & $\nu_{\tau}$ & 0 & 0 & 1 & 0 & 0 \\
\hline 14 & $\bar{\nu}_{\tau}$ & 0 & 0 & -1 & 0 & 0 \\
\hline 15 & $\mu^{-}$ & -1 & 0 & 0 & 0 & 1 \\
\hline 16 & $\mu^{+}$ & 1 & 0 & 0 & 0 & -1 \\
\hline 17 & $\nu_{\mu}$ & 0 & 0 & 0 & 0 & 1 \\
\hline 18 & $\bar{\nu}_{\mu}$ & 0 & 0 & 0 & 0 & -1 \\
\hline 19 & $e^{-}$ & -1 & 0 & 0 & 1 & 0 \\
\hline 20 & $e^{+}$ & 1 & 0 & 0 & -1 & 0 \\
\hline 21 & $\nu_{e}$ & 0 & 0 & 0 & 1 & 0 \\
\hline 22 & $\bar{\nu}_{e}$ & 0 & 0 & 0 & -1 & 0 \\
\hline
\end{tabular}

Table 1

Some Common Particles and Quantum Number Assignments

on, I will use the term "quantity" to refer to such process-invariant, or timeinvariant, properties of particles. In this paper I consider only conservation principles that involve process-invariant quantities. To fix some notation, the fact that particle $p$ carries $x$ units of quantity $q$ will be denoted by $q(p)=x$. For instance, if $C$ denotes electric charge, we have $C\left(e^{-}\right)=-1$.

A reaction conserves a quantity just in case the total sum of the quantity over the reagents is the same as the total sum over the products. For example, the reaction $p+p \rightarrow p+p+\pi^{0}$ conserves Baryon number, since the Baryon total of 
the reagents is $2 \times$ Baryon $\#(p)=2 \times 1$, and the Baryon total of the products is $2 \times$ Baryon $\#(p)+$ Baryon $\#\left(\pi^{0}\right)=2 \times 1+0$.

The conservation of the quantities electric charge, baryon number, tau number, electron number and muon number that are shown in Table 1 is part of the Standard Model that takes the quarks as building blocks of particles and was elaborated by particle physicists in decades of research $[10,2,41,22,3]$. For brevity, I will sometimes abbreviate these quantities as $C, B, T, E, M$.

A comment is in order regarding the current status of these conservation laws. Since the recent discovery that neutrinos have nonzero mass (1998-2000), there has been extensive activity in extending the Standard Model of particle physics on the basis of new experimental data [35,3]. Briefly, the nonzero mass of neutrinos permits quantum-mechanical effects that violate traditional conservation laws such as muon number. For instance, in "neutrino oscillation" a muon neutrino turns into a tau neutrino, which clearly violates muon number conservation. Particle theory implies that such violations occur very rarely, but nonetheless there seems to be sufficient evidence that they do occur in nature. The picture that seems to be emerging is that some of the conservation laws of the Standard Model such as muon or baryon number conservation are partial symmetries that hold only "approximately", that is with very rare exceptions. For example, the only reactions that may fail to conserve baryon number are so-called chiral anomalies [9]. There are complex current debates in particle theory about the best way to extend the Standard Model to account for these new phenomena. My aim in this paper is not to describe possible extensions of the Standard Model. (A textbook reference is [40].) Instead, I will focus on the pre-1998 Standard Model and the particle phenomena that it covers, where the quantities listed in Table 1 are universally conserved. It is possible to carry out the following analysis for a model with partial symmetries and approximate conservation laws. The results would be essentially the same, and the extra complications do not lead to new epistemological insights.

\subsection{Selection Rules and Local Underdetermination}

Let us see how the use of conservation principles can resolve local underdetermination. Suppose that other resources from physical theory, known conservation principles, etc., do not suffice to answer the question of how many pions can be produced in a collision of two protons. Suppose further that the answer must lie with conservation principles governing collisions of two protons. Now if we observe the reaction $p+p \rightarrow p+p+\pi^{0}$, we infer that whatever conservation principles govern collisions of two protons, the pion $\pi^{0}$ must carry 0 of any conserved quantity, because clearly the two protons on the left and on the right put the same weight into the conservation balance. But if the pion 
$\pi^{0}$ carries 0 of every conserved quantity, then two protons may produce any number of pions without violating a conservation law. Thus after observing one reaction such as $p+p \rightarrow p+p+\pi^{0}$ that produces pions, we can deduce that any number of pions can be produced in a collision of two protons - which is what current particle theory tells us [8, p.82].

So if one reaction is possible ( $\operatorname{such}$ as $p+p \rightarrow p+p+\pi^{0}$ ), then selection rules must permit other reactions as well (such as $p+p \rightarrow p+p+\pi^{0}+\pi^{0}$ ). Thus focusing on conservation principles allows us to infer that certain unobserved reactions are possible. For a given set of observed reactions $R$ there will be a set $R^{\prime}$ of reactions that are entailed by the observed data $R$ in the sense that any set of selection rules consistent with the reactions $R$ must also be consistent with the reactions $R^{\prime}$. I shall refer to the reactions $R^{\prime}$ entailed by a data set $R$ as the least generalization of $R$.

If our goal is to explain the nonoccurrence of unobserved reactions, we would seek a set of selection rules that rule out as many unobserved reactions as possible. Let us say that such a set of selection rules is maximally strict for the observed reactions $R$. In other words, a set of selection rules $Q$ is maximally strict for observed reactions $R$ just in case the reactions that conserve all quantities in $Q$ form the least generalization of $R$. The next proposition asserts that, for any set of observed reactions $R$, there are infinitely many maximally strict sets of conservation laws.

Proposition 1 Let $R$ be any set of observed reactions. Then there are infinitely many maximally strict sets of conservation laws, each of which allows exactly the least generalization of $R$.

Here are the intuitive reasons why the proposition holds. The key observation is that we can think of particle reactions that are allowed by a given set of selection rules as forming a linear space. For example, given two reactions $r$ and $r^{\prime}$ that are consistent with a given set of selection rules $Q$, we can "add" $r$ and $r^{\prime}$ to obtain the reaction $r+r^{\prime}$ that is also consistent with $Q$. To illustrate, both the neutron decay $n \rightarrow p+e^{-}+\bar{\nu}_{e}$ and muon decay $\mu^{+} \rightarrow e^{+}+\nu_{e}+\bar{\nu}_{\mu}$ conserve all standard quantities. Hence so does their sum, which is the collision $n+\mu^{+} \rightarrow$ $p+e^{-}+\bar{\nu}_{e}+e^{+}+\nu_{e}+\bar{\nu}_{\mu}$. The least generalization of a given set of reactions $R$ is the least linear space containing $R$; that is, the least generalization comprises the reactions that can be generated as linear combinations of reactions in $R$. Section 10 explains how reactions are represented as vectors.

How can we find a set of selection rules that is consistent with exactly the least generalization of the reactions $R$ ? The answer is to note that the set of quantities that are conserved in the reactions $R$ forms another linear space (technically, this linear space is the orthogonal complement of $R$ ). For example, given the standard model quantities Baryon Number and Electric Charge, 
we can define their sum Baryon-Charge to be the quantity that assigns to every particle the sum of its Baryon Number and its Electric Charge. To illustrate, the Baryon-Charge of the proton $p$ is $1+1=2$. It is not hard to see that Baryon-Charge is conserved in every reaction that conserves Baryon Number and Electric Charge. In other words, Baryon-Charge is redundant given Baryon\# and Electric Charge. In general, a linear combination of conserved quantities will also be conserved, but does not add any more constraints on particle reactions.

A maximally strict set of conservation laws is a set that contains as many irredundant laws as possible. Each law adds a constraint on how nature may behave, so the more irredundant laws a theory contains, the stricter it is. Given that linear combinations of quantities are redundant, what we seek is a maximal set of linearly independent quantities; in linear algebra terms, a basis for the space of quantities conserved in the reactions $R$. Figure 1 illustrates these facts. It is easy to see that the space of conserved quantities
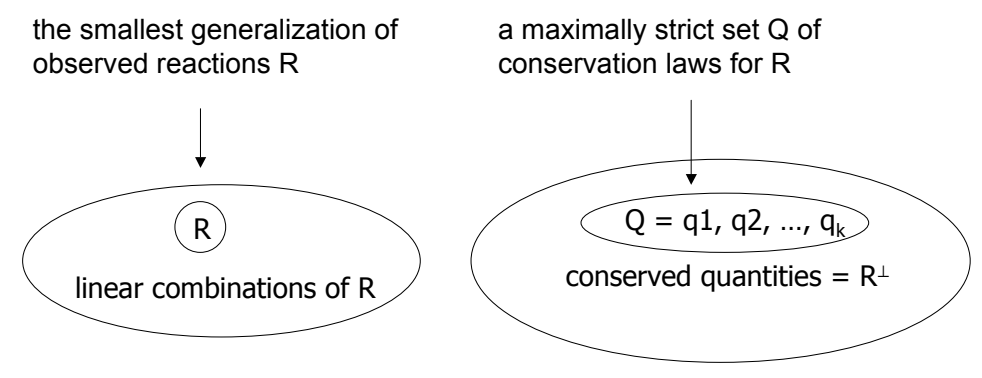

Fig. 1. The least generalization of observed reactions $R$ is to predict that all and only linear combinations of the data $R$ are possible. The set of quantities that are conserved in all the reactions $R$ forms a linear space, the orthogonal complement $R^{\perp}$. Any set of conservation laws $Q$ that corresponds to a basis for $R^{\perp}$ allows precisely the least possible generalization of the observed reactions $R$.

has infinitely many bases. For example, we can multiply Baryon Number by 2 to form the quantity $2 B$ defined by $2 B(p)=2 \times B(p)$. For instance, the proton carries two units of $2 \mathrm{~B}$ since it carries one of $B$. Now we can replace the conservation of Baryon Number $B$ by the conservation of $2 B$ and obtain a predictively equivalent theory that is consistent with exactly the same reactions. More formally, the set of quantities $\{2 B, M, E, T\}$ is predictively equivalent to the set $\{B, M, E, T\}$. Similarly, we could replace $B$ by $3 B, 4 B$, etc. Formal statements and proofs of these results may be found in [31].

Given the emphasis that physicists have placed on explaining what does not occur (cf. Section 2), our analysis so far suggests the following inductive rule for generalizing from observed data to a set of conservation laws: always adopt a set of conservation laws that are maximally strict. I refer to this rule as the maximally strict rule (MSR for short). 
There are two basic questions we may ask about the maximally strict rule. 1) the epistemologist's question: what sort of justification is there for the maximally strict rule, for example in terms of more general epistemic principles?

2) the naturalist's question: do maximally strict theories match what we find in scientific practice? I discuss these questions in the next section.

\section{Inferring Conservation Laws: Methodological Analysis and Com- parison With Practice}

This section examines the status of the maximally strict inference rule, first from the point of view of general inductive methodology, second in terms of agreement with scientific practice.

\subsection{Means-Ends Justification of the Maximally Strict Inference Rule}

In a previous article [31] I discussed the status of the maximally strict rule from the point of view of an inductive epistemology based on means-ends analysis. I review here the main results of the investigation of the MSR rule. The basic tenet of means-ends analysis is that good inference methods are those that attain the goals of inquiry. Means-ends epistemology examines a number of standards of empirical success, such as reliable convergence to the truth, fast convergence, and stable convergence $[16,30,13]$. To keep matters simple, let us consider two aims: reliable and stable convergence to a theory that makes correct predictions. The notion of reliable convergence to a correct theory stems from a Peircean vision of empirical success in which science may err in the short run, but corrects itself and eventually settles on the truth. Reichenbach's well-known vindication of induction is in this spirit: he showed that the posits of his straight rule come arbitrarily close to the true limiting relative frequency of an event, if that limit exists $[28,29]$. Putnam generalized Reichenbach's idea to develop a general theory of inductive inference $[24,26]$, which has grown into a mathematical subject known as Formal Learning Theory $[13,16,33]$.

Assume that some set of additive conservation laws is predictively equivalent for the totality of all reactions ever to be observed. Then the maximally strict rule is guaranteed to eventually arrive at a predictively adequate theory, and thus satisfies the Peirce-Reichenbach-Putnam ideal of empirical inquiry. This is because assuming that the possible particle reactions are correctly defined by a set of selection rules, the possible reactions form a linear space, and there is a finite subset $F$ such that all possible reactions are linear combinations of those in $F$. So as ever more particle reactions are observed, the MSR will 
eventually adopt a predictively adequate set of laws, after a sufficiently informative finite subset of reactions $R$ has been observed. As we saw in Section 2, a theory that makes correct predictions with respect to the actually occurring reactions need not be correct about which physical processes are possible in principle, because a physically process may never actually occur. But if we assume Gell-Mann's plenitude principle, a theory that is empirically adequate with respect to actually occurring reactions is correct about which physical processes are possible in principle. So under plentitude the maximally strict rule is guaranteed to converge to the truth about which reactions and possible and which are not. Instead of a full plenitude principle, it suffices to assume that a sufficiently informative finite subset of the physically possible reactions will eventually be observed (i.e., a basis for the set of physically possible reactions).

A venerable philosophical tradition supports the idea that stable belief is a significant epistemic good. Since Plato's Meno, philosophers have been familiar with the idea that stable true belief is better than unstable true belief. Epistemologists such as Sklar have advocated principles of "epistemic conservatism" [34]. Kuhn tells us that a major reason for conservatism in paradigm debates is the cost of changing scientific beliefs [18]. In this spirit, means-ends epistemologists, starting with a seminal paper of Putnam's [25], have examined inductive methods that minimize the number of times that they change their theories before settling on their final conjecture $[16,30]$.

It turns out that the means-ends criteria of reliable convergence to the right answer and minimizing theory changes single out the maximally strict rule. I record this fact in the following proposition.

Proposition 2 Assume that there is a set of selection rules that correctly predicts which processes among $n$ known particles occur. The maximally strict rule is the only inference rule that satisfies the following two aims:

(1) The rule is guaranteed to eventually settle on a set of conservation laws that correctly predicts which reactions can and cannot occur; and

(2) the rule changes its predictions at most $n$ times.

The demonstration is in [31, Sec.7]. This result warrants a few comments. (1) The maximally strict rule changes its mind only when its most recent conservation law theory is falsified (as neutrino oscillation falsifies the conservation of muon number, Sec. 3), so it is also the case that the MSR is the only rule whose predictions are falsified at most $n$ times (under the assumptions of the proposition). (2) Philosophers of science are familiar with the distinction between the predictive or empirical adequacy and the truth of a theory [39]. In our context, the distinction is between a theory that correctly predicts which processes occur and one that correctly indicates which processes 
are possible in principle; the distinction makes a difference just in case some possible process never occurs. As discussed in Section 2, Gell-Mann's Totalitarian principle rules this out, so with this plenitude principle as an additional premise, Proposition 2 entails that the MSR will converge to the truth about which reactions are physically possible. (3) The proposition illustrates the philosophically important point that criteria of inductive success can strongly constrain empirical conjectures in the short run. Elsewhere I have shown that the same inductive goals select the generalization "all emeralds are green" over all "emeralds are grue" in a Goodmanian Riddle of Induction [30].

Thus it appears that means-ends analysis matches scientists' methodological practice, in the sense that means-ends analysis directs inductive inquiry to adopt maximally strict conservation law theories, which corresponds to the principle of explaining as many unobserved reactions as possible. In the next sections I examine how the inferences of the maximally strict rule compare with the theories physicists have developed in response to the currently available data. There are several steps in this project. First, to collect from the literature a suitable set of known reactions for applying the maximally strict method. Second, to compute a maximally strict set of conservation principles. Third, to determine whether the laws $C, B, M, E, T$ shown in Table 1 form a maximally strict set for the observed reactions.

\subsection{Collecting Particle Reaction Data}

To facilitate computational analysis, we need to supply reaction data in electronic format. Hundreds if not thousands of reactions are stored in databases such as HEPbase compiled by the Durham database group [12]. However, these sources do not readily provide a complete data set, but only information about one specific reaction at a time. We can find a representative and manageable subset of reactions as follows. It is a basic fact about the nature of evidence that independent datapoints provide more information than interdependent ones. In our case, the relevant notion of independence is linear independence. Specifically, it is easy to see that if we observe reactions $r_{1}, . . r_{k}$ and then a new reaction $r_{k+1}$ that is a linear combination of the set $\left\{r_{1}, \ldots, r_{k}\right\}$, then any conservation theory consistent with the observations $\left\{r_{1}, . ., r_{k}\right\}$ is also consistent with $\left\{r_{1}, . ., r_{k+1}\right\}$. In other words, a new linearly dependent reaction $r_{k+1}$ was already considered possible. So the most informative data are those comprising linearly independent reactions. To find linearly independent reactions, I take recourse to background knowledge - namely that particle reactions satisfy the principles of Special Relativity. The connection between Special Relativity and linearly independent reactions is as follows.

Lemma 3 Let $p_{1}, \ldots, p_{u}$ be particles with decay modes, and let $d_{j}$ be a decay 
mode for each particle $p_{j}$. Then assuming conservation of energy and momentum as defined in Special Relativity, the set of decays $\left\{d_{j}: 1 \leq j \leq u\right\}$ is linearly independent.

The proof is in [31, Th.5]. Lemma 3 can be applied to find an informative set of known particle reactions as follows: For every particle that has a decay mode, we select a decay (typically the most frequent decay mode). For example, for the upsilon1S particle whose symbol is $\Upsilon(1 S)$, I include the decay mode $\Upsilon(1 S) \rightarrow \mu^{+}+\mu^{-}$. The decay modes are listed in the Annual Review of Particle Physics, an authoritative publication that summarizes the current state of knowledge in the field every year

[6]. In all, my data set includes 205 observed reactions, 199 of which are decays listed in the Review. The data set includes a decay for each of the 182 particles with a decay mode listed. This comprises all particles except for $\gamma, e^{-}, e^{+}, p, \bar{p}, \nu_{e}, \bar{\nu}_{e}, \nu_{\mu}, \bar{\nu}_{\mu}, \nu_{\tau}, \bar{\nu}_{\tau} \cdot{ }^{4}$ Typically, the data include the most probable decay listed in the Review of Particle Physics. The additional reactions are important processes listed in textbooks.

The next step is to convert the reactions into their vector representation. This is done by a conversion utility, which produces a set of 205 vectors of dimension 182. Inspection of this set of vectors confirms that the 182 decays are linearly independent, as Lemma 3 entails.

\subsection{Applying the Maximally Strict Inference Rule to Current Particle Data}

After translating current particle data into vector format, we can run a program that produces a maximally strict set of conservation principles for this data. ${ }^{5}$ As we saw in Section 3, finding a maximally strict theory is equivalent to finding a basis for the orthogonal complement of the given reaction data. Although for a human this is a formidable task, fortunately it is a well-studied problem in computational linear algebra [15], and efficient programs for solving it are available that can analyze even an extensive set of reaction data in minutes. The next step is to compare the set of conservation principles produced by the program with the Standard Model laws $C, B, M, E, T$. With the aid of the computer, it is simple to check that the two sets of laws span the same linear space and hence are empirically equivalent, that is, consistent with

\footnotetext{
4 We did not include all the different resonances for a type of particle. This is partly because the Review does not list all resonances as "solidly confirmed". Also, while different resonances differ in mass, they do not behave differently with respect to the additive quantum numbers under consideration in this paper. The complete particle database is posted in Excel format at [32].

5 A trace of a program run is posted at [32].
} 
exactly the same reactions. This computation establishes that the combined laws $C, B, M, E, T$ form a maximally strict theory for the current data.

Finding 1 Let $D$ be the particle reaction data described in Section 4.2. The combination of laws asserting the conservation of electric charge, baryon number, tau number, electron number and muon number, forms a maximally strict set of conservation laws for the reaction data $D$.

The finding answers the naturalist's question in the affirmative: The predictions made by the laws we find in the Standard Model are exactly those mandated by the maximally strict inference rule. According to Proposition 1, there are infinitely many sets of conservation laws that are empirically equivalent to the standard set - any basis spanning the same linear space will do. So even if local underdetermination is resolved by the directive to make maximally strict predictions, global underdetermination arises because there are many sets of laws that make the same (maximally strict) predictions. Among the infinitely many selection rules that make the same predictions as the set $C, B, M, E, T$, is there something special about this set?

\section{Underdetermination and Matter vs. Antimatter}

Naturally this question occupied particle physicists. Feynman thought the answer would lie in the fact that quantities such as Baryon number would turn out to relate to physical phenomena other than as a conserved quantity: "If charge is the source of a field, and baryon number does the same things in other respects it ought to be the source of a field too. Too bad that so far it does not seem to be, it is possible, but we do not know enough to be sure" [7, p.67]. Omnes devotes a chapter to deriving selection rules in his graduate text on particle physics. He gives "once and for all" a procedure for assigning these numbers [23, Ch.2]. He does not address the question of whether his procedure uniquely determines a set of quantum numbers, although he comments that various aspects of the number assignments are arbitrary. Williams remarks, commenting specifically on the conservation of lepton number (the sum $M+$ $E+T)$ : "this lepton number conservation is arbitrary and has no basis in more fundamental ideas" [41, p.285].

One of the main considerations that led physicists to these laws is that they correspond to ontological categories that find an independent interpretation and support in the Standard Model that takes the quarks as building blocks of particles. The term family conservation law for the conservation of baryon, electron, muon, and tau number reflects the connection between selection rules and particle ontology [41]. The idea that natural laws have a special connection to ontology has a long standing in philosophy; often the idea is expressed by the 
thesis that natural laws refer to fundamental ontological categories or natural kinds $[17$, p.6]. In this section and the next I pursue the connection between conservation laws and ontology to see how it affects the underdetermination of conservation principles.

The four quantities Baryon\#, Electron\#, Muon\#, Tau\# illustrated in Table 1 have the following interpretation in terms of particle families. First, the particle world is divided into Baryons and non-Baryons. Every non-Baryon receives Baryon\# 0. Each Baryon that is regular matter is assigned Baryon\# 1 (e.g., the proton $p$ ); each Baryon that is anti-matter is assigned Baryon\# -1, and usually its symbolic name is marked by an overline, like for instance the antiproton $\bar{p}$. Similarly Electron\# represents the Electron family, which comprises the particles $e^{-}, e^{+}, v_{e}, \bar{\nu}_{e}$ : all other particles receive Electron\# 0, the two electron type particles $e^{-}$and $\nu_{e}$ Electron\# 1, and the antiparticles $e^{+}$ and $\bar{\nu}_{e}$ receive Electron\# -1, as illustrated in Table 1. Muon Number similarly represents the muon particle family $\mu, \bar{\mu}, \nu_{\mu}, \bar{\nu}_{\mu}$ and Tau\# the tau particle family $\tau, \bar{\tau}, \nu_{\tau}, \bar{\nu}_{\tau}$.

Experiments with our inference program show that even though different runs produce different conserved quantities, they all reproduce the matter/antimatter distinction in the sense that if a particle $p$ is assigned $x$ units of a quantity, its antiparticle $\bar{p}$ is assigned $-x$. Thus particles that are their own antiparticles, like the photon, are assigned 0 of every quantity. Let us say that a set of quantities $Q$ respects the matter/antimatter division if for every quantity $q$ in $Q$, if $q$ assigns $x$ to particle $p$ (i.e., $q(p)=x$ ), then $q$ assigns $-x$ to the antiparticle $\bar{p}$ (i.e., $q(\bar{p})=-x$ ). Table 1 illustrates that the standard model selection rules respect the matter/antimatter division. The next proposition shows that the invariance of the matter-antimatter division with respect to different maximally strict sets of conservation rules is no accident.

Proposition 4 Let $Q, Q^{\prime}$ be any two predictively equivalent combinations of laws, with associated quantities. If all the quantities in $Q$ respect the matter/antimatter division, then so do all the quantities in $Q^{\prime}$. It follows that any combination of additive conservation laws that is predictively equivalent to the conservation of electric charge, baryon number, tau number, electron number and muon number respects the matter/antimatter division.

The proof is in Section 10. Roughly, the proposition holds because if $Q$ and $Q^{\prime}$ are predictively equivalent sets of conservation laws, then the quantities associated with them respectively form a basis for the same linear space. So all quantities associated with $Q^{\prime}$ can be expressed as a linear combination of the quantities in $Q$; that expression must respect the matter/antimatter division if the quantities in $Q$ do.

The proposition addresses both global and local underdetermination. For global 
underdetermination, consider the set of all reactions ever to occur in the course of nature; denote this set by Total. If this set is consistent with the matter/antimatter division, in the sense that some conservation theory $Q$ permits exactly the reactions in Total that respects this division, then all such conservation theories respect this division. In other words, the total reaction phenomena Total determine the matter/antimatter division. For local underdetermination, suppose we have obtained particle accelerator data $D$, and there is some conservation theory $Q$ that incorporates the matter/antimatter division and is maximally strict for $D$. Then the proposition implies that all other maximally strict conservation theories for $D$ respect the matter/antimatter division. In other words, the reaction data $D$ and the maximally strict rule together determine the matter/antimatter division. So such aspects of particle ontology as which particles are their own antiparticles and which particles act like antiparticles of others can be recovered from the reaction data alone without recourse to other considerations or a general ontological model (such as the Standard Model).

The result of this section shows a subtle relationship between the contingent and the necessary facts about nature. It does not assert as an a priori truth that there must be a fundamental ontological split between matter and antimatter. Rather, it says that if it is possible to have any empirically adequate conservation theory that incorporates this division, then all empirically adequate conservation theories must incorporate this division. If the physicists' current theory is empirically adequate, then as a matter of contingent fact there is an empirically adequate conservation theory that respects the matter/antimatter division. Given this contingent fact, it follows from the proposition that necessarily all empirically adequate conservation theories incorporate this cleavage. In terms of the metaphor of ontological categories as joints of nature, the particular joint corresponding to the matter/antimatter division need not have been part of nature, but if it is, then all predictively adequate conservation theories must include it.

By measuring the mass, charge, and spin of particles it is straightforward to determine which are antiparticles of each other. The point of this section is therefore not that we can determine the particle/antiparticle division by some measurement, but that reaction data alone, without further measurements, determine many aspects of this division (e.g., which particles are their own antiparticles). In the next section we examine the determination, from reaction data alone, of particle families. 


\section{Underdetermination and Particle Families}

This section examines the underdetermination of particle taxonomy by reaction data. I establish two new theorems in linear algebra showing that a division of particles into disjoint families is, under quite general conditions, uniquely determined by reaction data. Then I apply these results to arrive at the main finding of the case study in this paper: the available reaction data did uniquely determine the Standard Model particle families comprising the baryons and the three lepton generations tau, electron and muon.

\subsection{Do Particle Reaction Data Determine Particle Families?}

In contrast with the matter/antimatter distinction, the partition of particles into baryons, muons, electrons and tau types is not necesssary for a predictively adequate conservation theory: it is easy to produce theories that are equivalent to the standard laws but do not incorporate these distinctions. For a very simple example, suppose we form a new quantity $q$ defined as the vector sum of baryon number and muon number, such that each particle $p$ carries an amount of $q$ the sum of its baryon and muon numbers (in symbols, $q(p)=B(p)+M(p))$. Now replace baryon number by $q$, which results in the law set $\{C, q, M, E, T\}$. This set is empirically equivalent to the standard rules, in that a reaction $r$ conserves all quantities just in case $r$ conserves all quantities in the set; but no conservation law in the new set corresponds to the family of baryons. A skeptic with nominalist leanings (sometimes called a "conventionalist" [4, Sec.4]) may point to such examples as evidence that ontological categories such as "baryon" have no reality in nature, but are imposed by us to explain the particle reaction phenomena, while equally good (i.e., predictively equivalent) explanations are available that do not employ these categories.

There is a reply to the nominalist: what is better about the standard family laws compared to the gerrymandered ones is that they divide the particle world into disjoint categories. Laws based on disjoint categories have several virtues. (1) They satisfy the intuition that the laws of nature should be based on fundamental distinctions among the objects in the world. (2) They can be interpreted as asserting that particles of one kind do not transform into particles of another, which seems physically meaningful. (3) They define ontological categories that may point to and receive support from independent ontologies. For example, in the quark model of particles the baryons turn out to be exactly the particles composed of three quarks.

Fair enough, says the nominalist. But surely we can imagine an alternative 
set of disjoint categories, different from the baryon, muon etc. categories, with corresponding conservation laws that are predictively equivalent to the standard selection rules? I will show that the answer is no: What the nominalist says he can imagine is in fact logically impossible.

Say that a particle $p$ carries a quantity $q$ if $q(p) \neq 0$. A set of quantum numbers $\left\{q_{1}, \ldots, q_{j}\right\}$ forms a family set if no particle carries two quantities; formally, if $q_{i}(p)=0$ whenever $q_{j}(p) \neq 0$, for all $i \neq j$. Given a family set of quantities, the carriers of the quantities form disjoint particle families. The standard quantities $\{$ Baryon\#, Muon\#, Electron\#, Tau\#\} form a family set. For example, the proton carries 1 unit of baryon number, but 0 of the muon, electron and tau numbers. In the debate with the nominalist, the issue is how many family sets in addition to the standard particle families there might be. The surprising answer is that if there is any predictively adequate family set, then all predictively adequate family law sets define the same particle ontology.

Theorem 5 Let $Q, Q^{\prime}$ be any two predictively equivalent combinations of laws, with associated quantities. If both $Q$ and $Q^{\prime}$ are family sets, then they define the same particle families as carriers of their quantities. It follows that if $Q^{\prime}$ is any family set of laws that is predictively equivalent to the conservation of baryon number, tau number, electron number and muon number, then $Q^{\prime}$ defines the same particle families as these laws (namely the baryons and the three lepton generations tau, electron and muon).

The proof is in Section 10. The basic argument runs as follows. Consider two conservation theories, $Q, Q^{\prime}$ that are predictively equivalent (i.e., consistent with exactly the same reactions) such that $Q$ and $Q^{\prime}$ are family sets. The structure of vector spaces requires that it must be possible to translate $Q$ into $Q^{\prime}$. However, the proof shows that if one family set $Q$ can be translated into another $Q^{\prime}$, then the particle families corresponding to $Q$ must be the same as those for $Q^{\prime}$.

The theorem does not show that the exact quantities are uniquely determined. Recall from Section 3.2 that we may multiply Baryon\# by 2 to form the vector $2 B$ defined by $2 B(p)=2 \times B(p)$. For instance, the proton carries two units of $2 \mathrm{~B}$ since it carries one of $B$. The set $\{2 B, M, E, T\}$ is predictively equivalent to the set $\{B, M, E, T\}$, but this does not contradict the theorem because the carriers of Baryon\# are exactly the same as those of $2 B$, namely the Baryons.

The proposition addresses both global and local underdetermination. For global underdetermination, consider the set of all reactions ever to occur in the course of nature; denote this set by Total. If this set is predicted correctly by a family law set $Q$, in the sense that $Q$ permits exactly the reactions in Total, then all other family law sets $Q^{\prime}$ that permit exactly the reactions in Total define 
the same particle families. In other words, the total reaction phenomena Total determine a unique set of particle families. For local underdetermination, suppose we have particle accelerator data $D$, and there is some family law set $Q$ that is maximally strict for $D$. Then the proposition implies that all other maximally strict family conservation theories for $D$ define the same families. In other words, the reaction data $D$ and the maximally strict rule together determine the division of particles into disjoint families, without recourse to other considerations or a more general ontological model (such as the Standard Model).

The theorem establishes another subtle interplay between the contingent and the necessary. It need not be the case that there is any partition of particles into families that corresponds to empirically adequate conservation laws. But given that there is such a partition, it is unique. In terms of the joints metaphor, there need not have been any empirically adequate way of carving up nature, but given that there is one, the cuts are uniquely determined.

As striking as this observation is, there is still a flaw in our reponse to the nominalist: we do not in fact have an empirically adequate family set of conservation laws. For the set of empirically adequate laws comprises not just the family set $\{B, M, E, T\}$ but also the conservation of electric charge. And the complete set of laws $\{C, B, M, E, T\}$ is not a family set because the carriers of electric charge include, for instance, many baryons, such as the proton. However, charge has a special relationship to the other laws that is relevant: charged particles are spread through each of the other types such that (1) some, but not all particles of each type are charged, and (2) for a given type some, but not all charged particles belong to that type. For example, the proton is charged and a baryon, whereas the neutron is neutral and a baryon. Formally, let us say that a quantity $q$ is logically independent of another set of quantities $\left\{q_{1}, . ., q_{k}\right\}$ if for every $q_{i}$ we have that (1) some, but not all carriers of $q$ are also carriers of $q_{i}$, and (2) some, but not all carriers of $q_{i}$ are also carriers of $q$. Then electric charge $C$ is logically independent of the family conservation laws $\{B, M, E, T\}$. The next theorem shows the point of this observation: given an empirically adequate set of laws consisting of a family set and a logically independent quantity, the carriers of the family set are uniquely determined.

Theorem 6 Let $Q$ be a family set of laws, with at least two associated quantities $\left\{q_{1}, . ., q_{m}\right\}$, and consider a quantity $q$ that is logically independent of the quantities $\left\{q_{1}, . ., q_{m}\right\}$ associated with $Q$. Let $Q^{\prime}$ be any other family set of laws, with associated quantities $\left\{q_{1}^{\prime}, \ldots, q_{m}^{\prime}\right\}$, such that the conservation of all quantities $\left\{q, q_{1}^{\prime}, \ldots, q_{m}^{\prime}\right\}$ permits exactly the same reactions as the conservation of all quantities $\left\{q, q_{1}, . ., q_{m}\right\}$. Then $Q$ and $Q^{\prime}$ define the same particle families as carriers of their quantities. It follows that if $Q^{\prime}$ is any family set of laws that together with electric charge is predictively equivalent to the conservation of 
electric charge, baryon number, tau number, electron number and muon number, then $Q^{\prime}$ defines the same particle families as baryon number, tau number, electron number and muon number (namely the baryons and the three lepton generations tau, electron and muon).

This result generalizes the scenario envisioned in Theorem 5 to a more complex scenario, with a similar moral. Assume that the conservation of a certain quantity $q$, like electric charge, is given by background knowledge. If some combination of $q$ and a logically independent family law set $Q$ permits exactly the set of all reactions ever to occur in nature, then the total reaction phenomena uniquely determine the particle families in $Q$. Suppose particle reaction data comprise a finite data set $D$ such that some combination of $q$ and a logically independent family law set $Q$ are maximally strict for $D$. Then the data $D$ and the maximally strict rule together determine the particle families in $Q$.

\subsection{The Reaction Data Determine the Standard Particle Families}

Our results so far show how the logical structure of additive conservation laws resolves the underdetermination of theory by evidence in general; let us now consider how they apply to our case study. Consider the reaction data that were consistent with the conservation of Charge, Baryon\#, Tau\#, Electron\#, and Muon\# and led to the adoption of these conservation laws; this data includes the data set $D$ described in Section 4.2. As Feynman pointed out (cf. Section 5), charge is different from the other additive conservation laws in that it is the source of a field and relates to other dynamic phenomena. Thus we can view the charge of a particle as being determined independently. Moreover, the law of the conservation of electric charge was well established in physics long before the study of subatomic particles. So for the project of finding new conserved quantities for the subatomic world, the conservation of the $C$ quantity can be taken as given.

The next finding records that if we take the conservation of electric charge as our starting point, the actual reaction data described previously uniquely determine the $\{B, M, E, T\}$ families.

Finding 2 Let $D$ be the particle reaction data described in Section 4.2. Let $Q$ be any family set of conservation laws such that the combination of $Q$ and electric charge is maximally strict for the reaction data $D$ from particle physics. Then

(1) $Q$ respects the matter/antimatter division of elementary particles, and

(2) $Q$ defines the same particle families as the standard model laws, namely baryons and the three lepton generations tau, electron and muon. 
Figure 2 illustrates this result. The finding holds for the following reasons.

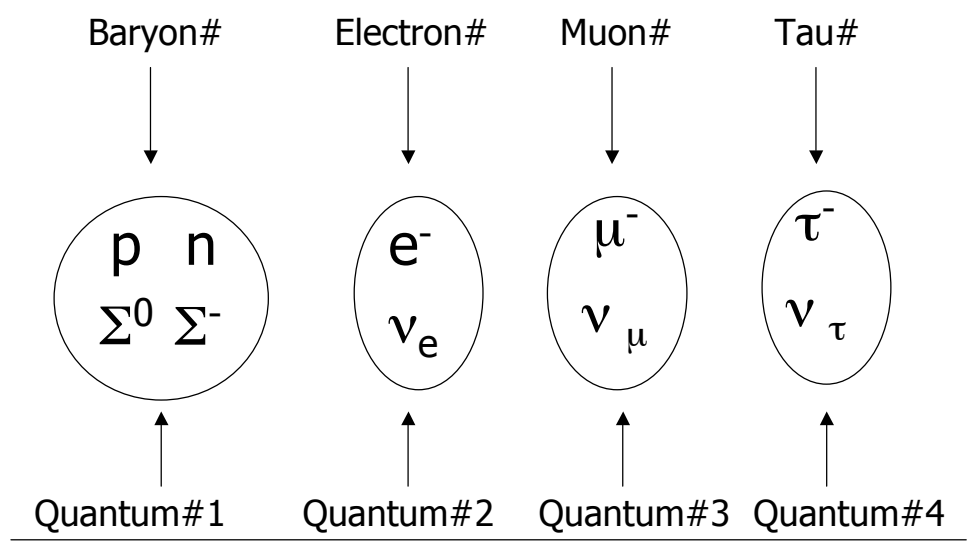

Any alternative family set with 4 quantum numbers

Fig. 2. Given the law of conservation of electric charge, any maximally strict set of laws that partitions the particle world into disjoint categories employs the same categories as the standard family conservation laws.

From Finding 1 we know that the laws asserting the conservation of electric charge, baryon number, tau number, electron number and muon number, form a maximally strict set of conservation laws for the reactions $D$. So if $Q$ plus electric charge is maximally strict for the reaction data $D$, then $Q$ plus electric charge makes exactly the same predictions as the set of laws corresponding to the conservation of electric charge, baryon number, tau number, electron number and muon number. So Proposition 4 implies that $Q$ respects the matter/antimatter division of elementary particles. Also, Theorem 6 implies that the two sets of laws must respectively define the same particle families.

From the point of view of local underdetermination and inductive inference, Theorem 6 gives strong guidance to finding a maximally strict conservation theory: it suggests to start with electric charge and its conservation as given, and then search for family laws that 1) correspond to disjoint ontological categories, and 2) in combination with electric charge yield a maximally strict theory. It turns out that the parsimony of a set of selection rules guides an inference method towards a simple ontology.

\section{Discovering Particle Families through Parsimony}

So far we have seen a special status for the conservation of electric charge, baryon number, tau number, electron number and muon number: these laws correspond to the uniquely simplest ontology consistent with a maximally 
strict conservation theory. How can scientists discover laws with this special status given a finite set of reaction data observed so far?

One way to implement the search for maximally strict family conservation laws computationally is to seek a set of maximally strict set of conservation laws such that for each quantity, a minimal number of particles carry that quantity. Mathematically, we seek vectors with many 0s. My program searches for a maximally strict conservation theory that includes electric charge and minimizes the sum of the absolute values of the amounts carried by all particles. Formally, for a set of quantities $q_{1}, . ., q_{k}$ ascribed to $n$ particles, the measure to be maximized is $P\left(\left\{q_{1}, \ldots, q_{k}\right\}\right)={ }_{d f}-\sum_{j=1}^{k} \sum_{i=1}^{n}\left|q_{j}(i)\right|$. For instance, the proton contributes $1+1=2$ to this sum for carrying 1 unit of charge and 1 of baryon number. Its antiparticle $\bar{p}$ contributes $|-1|+|-1|=2$, the same amount. The sum of absolute values is a natural measure of the parsimony of a set of conservation laws that has previously been used in the literature [37,36]. Minimizing this sum leads the program to rediscover the Standard Model family laws, which establishes the following finding.

Finding 3 The combination of the conservation of electric charge, baryon number, tau number, electron number and muon number is maximally parsimonious compared to other sets of laws that include the electric charge $C$. That is, if $Q=\left\{C, q_{1}, . ., q_{4}\right\}$ is predictively equivalent to $\{C, B, M, E, T\}$, then the sum of (absolute) values in $Q$ is at least as great as the sum of values in $\{C, B, M, E, T\}$.

There is an analytic explanation of this finding: Using Theorem 6 and Proposition 4 , one can show that if there is a family set $F$ like the standard laws that assigns only magnitudes of 0,1 , or -1 , then this family set is maximally parsimonious, and all maximally parsimonious predictively equivalent sets of selection rules define the same particle ontology as $F$. In other words, parsimony of selection rules is a guide to the maximally simple division of particles into families.

I conclude my case study by comparing my algorithmic derivation of selection rules and particle families with the historical progress of particle theory. One difference with the historical development is that often ontological categories were epistemically prior to reaction data. For example, if one arranges elementary particles by mass, there is a large gap between the lightest baryon (the proton) and the heaviest non-baryon particle (baryon number was also called "heavy particle number"). So the category of baryons suggests itself independently of reaction data; the baryon number quantity can be derived from this category (as described in Section 5), and then its conservation checked against the available reaction data. This is the kind of procedure that Feynman referred to as a "quick way of guessing at the laws of nature" [7, p.67]. 
In contrast, in the perspective of my analysis particle families are epistemically posterior to reaction data, because they are discovered by analyzing the reaction data, not posited first and then checked against the data. The historical reliance on considerations and measurements in addition to reaction data, such as the masses of particles, would suggest that particle families cannot be discovered from reaction data alone, but must be derived from other sources. The surprising and novel result of my analysis is that this is not so; this new insight is what motivates developing a method that begins with reaction data to find particle families.

The connection between particle ontology and particle dynamics goes substantially beyond the connection between particle families and selection rules that I have studied in this paper. For example, dynamically relevant properties of particles include their masses, spins, whether they are fermions, bosons etc. A simple illustration is the fact that the conservation of energy together with the masses of the particles implies constraints on what reactions are possible (cf. Lemma 3). A rewarding extension of my study would be to consider how further aspects of ontology and classes of conservation laws other than selection rules mutually constrain each other; I leave this for another occasion.

\section{Discussion: Induction, Laws, Simplicity, and Natural Kinds in Particle Physics}

Our analysis has touched on some of the great themes in the philosophy of science as they pertain to conservation laws in particle physics. In this section I take a broader perspective and place my findings in the context of more general discussions in the philosophy of science. To recapitulate, we found that the Standard Model of particle physics features the set of conservation laws that yield the best generalizations about particle dynamics and the simplest particle taxonomy with the smallest number of particle families.

What kind of Simplicity? I have employed two measures of the simplicity of conservation laws: Parsimony and ontological simplicity. We measured the parsimony of a law in terms of the (absolute) magnitude of the quantities the law assigns - the smaller the better. The parsimony of a set of laws is the sum of their individual parsimonies. Thus it is more parsimonious to assign the electron a charge of -1 and the proton a charge of +1 , rather than -2 respectively +2 even if we could replace the charge number $C$ by its double $2 C$ without loss of predictive accuracy. If we think in terms of a table such as that in Table 1 representing all the conservation laws, our notion of parsimony measures the size of the table. The idea that hypotheses whose representation is smaller are to be preferred has been widely investigated and applied in statistics and computer science, under the rubric of "minimum description 
length" [21, Ch.28.3].

The operative notion of ontological simplicity in my analysis has basically two aspects: first, number - to employ as few ontological categories as possible (in our case four not counting charge), and second, overlap - to find as many particle families as possible that are disjoint, that is, categories whose boundaries do not overlap. In Section 7 I established an analytic connection between parsimony and ontological simplicity: in our domain, parsimony is a guide to ontological simplicity. However, in terms of scientific significance, parsimony pales in comparison to ontological simplicity in particle physics. For example, the discovery of a muon-flavored neutrino introduced a fundamental new category in the particle world and was much debated [8]. In contrast, increasing the magnitude of quantum numbers (e.g., taking the basic unit of electric charge to be 2 rather than 1) would be just a different, albeit akward, way of describing the same physical reality.

Simplicity, Truth, and Saving the Phenomena. We have seen that when it comes to conservation theories, ontological simplicity is a feature that distinguishes among all the predictively adequate conservation theories. There is a realist and an instrumentalist way of looking at this finding. The realist could view it as an example of how simplicity can guide us to the truth even when saving the phenomena alone does not. The instrumentalist can reply that my case study illustrates how much value scientists place on ontological simplicity. But this does not establish a connection between simplicity and truth, but just a connection between simplicity and the theories we value. However, both sides can agree that it is remarkable that a fairly simple approach - Feynman's "quick ways of guessing at the laws of nature" - should be as predictively powerful as it is.

Lewis on Laws, Kinds and Simplicity. David Lewis uses the term "natural property" for the properties that "physicists discover in the course of discovering laws" [20, p.365]. As the distinction between natural kinds and natural properties is not important for my purposes, I will mostly follow Lewis in referring to natural properties. Many philosophers have noted connections between induction, laws and natural kinds or properties. Quine asked "what tends to confirm an induction?" [27, p.155] and held that "the answer lies in similarity" [27, p.157], "which is immediately definable in terms of kind; for, things are similar when they are two of a kind". Goodman's thesis that valid generalizations are the law-like ones is well-known. He also suggests that law-likeness involves natural kinds [11]. A connection between natural laws and natural kinds has been widely accepted by philosphers after Goodman. Kornblith encapsulates the view as follows $[17$, p.6].

On the account of science which began to emerge from these authors [Putnam, Boyd, Field and a "host of others"], it is the business of science to 
discover the real causal structure of the world; what this means, in a word, is the discovery of natural kinds and the causal relationships among them.

In his book "Counterfactuals", David Lewis' account of natural laws is, roughly, that a natural law is a member of the system of true generalizations that makes an ideal trade-off between simplicity and information content [19]. Our case study suggests a promising synthesis of Lewis' analysis with the traditional connection between laws and natural kinds: if a key ingredient of the simplicity of a system of generalizations is ontological simplicity, then Lewis' account of laws makes a connection with ontology. Let me elaborate this idea.

Suppose we add to Lewis' account of laws the thesis that an important part of the simplicity of a system of generalizations is ontological simplicity. Specifically, consider the following two theses:

(1) A law is a member of the simplest system of empirically adequate generalizations, where ontological simplicity is an important aspect of simplicity.

(2) A natural property/kind is one that appears in the ontologically simplest system of empirically adequate generalizations.

Our case study illustrates both theses. In particular for 2), we saw that the standard particle families are the ones that correspond to the ontologically simplest maximally strict set of conservation laws. The two theses imply a connection between laws and natural properties or kinds. For by Thesis 1), laws will tend to to be ontologically simple empirically adequate generalizations. And by Thesis 2), ontologically simple empirically adequate generalizations involve natural properties. If the maximally simple system $S$ of empirically adequate generalizations is also the ontologically simplest one, as in our case study, then it follows from 1) and 2) that the properties appearing in $S$ are also natural properties. Figure 3 illustrates these connections.

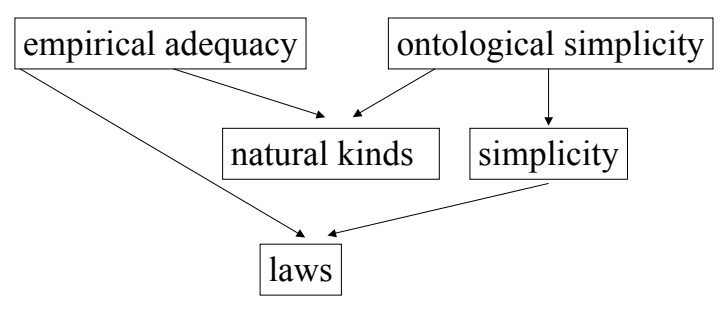

Fig. 3. To illustrate the thesis that natural kinds are those that appear in the ontologically simplest empirically adequate system of generalizations. Ontological simplicity is a component of simplicity, which connects Lewis' simplicity-based account of natural laws with ontology. Our case study shows that the four Standard Model particle families Baryon, Electron, Muon, and Tau correspond to the ontologically simplest system of conservation laws for the given particle reaction data.

It is instructive to contrast the two theses I stated above with Lewis' analy- 
sis of natural law. First, my analysis does not involve the trade-off between simplicity and information content envisioned in Lewis' theory. As far as conservation laws for particle reactions go, the trade-off does not arise because all systems of selection rules make complete predictions about which reactions are possible and which are not, and thus all have maximal information content for this domain. Lewis would presumably respond that this is a special case. Even though in other scientific domains we may well find a tension between obtaining a simple theory and making many predictions, there is an important point to be learned from our case study: research takes place within the context of definite scientific questions. The goal of scientific research is not a general quest for interesting regularities, but to answer specific questions, such as what particle reactions are possible. At a minimum scientific theories have to provide enough information content to answer the questions that prompted inquiry in the first place. Thus the context of the research goals constrains the acceptable trade-offs between simplicity and information content.

In a later article "New Work for a Theory of Universals", Lewis adds ontological considerations to his account of laws: for a given axiom set representing a system of generalizations, he stipulates that the primitive vocabulary of the axioms must refer only to natural properties [20, p.368]. Lewis' suggestion seems to assume a notion of natural property that is independent of empirical adequacy and of simplicity, and uses this independent notion to restrict the range of systems of generalizations that may count as laws. My proposal in contrast defines both the concept of law and that of natural property in terms of empirical adequacy, simplicity, and ontological simplicity.

On both views, "laws will tend to be regularities involving natural properties" and "the scientific investigation of laws and of natural properties is a package deal" [20, p.368]. Both views also agree that "in putting forward as comprehensive theories that recognise only a limited range of natural properties, physics proposes inventories of the natural properties instantiated in our world" [20, p.364]. My approach has an important advantage for understanding scientific discovery: Lewis' account does not address how scientists should go about finding natural properties (what Daly terms the "epistemological question" $[4$, Sec.2]), whereas on my proposal scientists can find natural properties by seeking a predictively adequate theory that is ontologically simple, as shown in Section 7. In our case study, the difference is that while Lewis' account explains why the conservation of baryon, muon, electron and tau number count as laws of nature given the natural particle families comprising baryons, muon-type particles, electron-type particles and tau-type particles, his account does not entail that physicists may go about discovering these categories by pursuing ontologically simple laws as they have done. 


\section{Conclusion}

Many physicists and philosophers have emphasized the importance of conservation laws. In his address as president of the PSA, John Earman said that

Philosophers of science have barely scratched the surface of the topic of laws, symmetries, and symmetry breaking. What I find most attractive about this topic is that it brings into fruitful interaction issues from metaphysics, from mathematics and physics, from the philosophy of scientific methodology, and from foundations of physics. [5, p.1240].

My case study is an example of the rich interaction that Earman describes. I examined some of the newer conservation laws that particle physicists introduced for the realm of subatomic particles. I considered additive conservation laws that assign an amount of some quantity $q$ to each particle, and are satisfied in a reaction if the sum total of $q$ among the reagents is the same as the sum total among the products. An example of a classical additive conservation law is the conservation of electric charge; a well-known new conservation law is the conservation of baryon number. The search for conservation laws has shown how the available evidence can help science find the truth despite concerns about the underdetermination of theory by evidence. A plenitude principle - Gell-Mann's "totalitarian principle" - asserts that the course of inquiry is sufficient to eventually bring into actuality all particle interactions that are physically possible, at least given enough experimental attention and resources. Conversely, if a process fails to be realized experimentally, there should be a conservation law that explains its nonoccurrence. This leads to a principle for inductive inference: When given a list of reaction data, find conservation laws that explain the absence of as many unobserved processes as possible. The question arises whether the laws that physicists actually adopted conform to this principle. With the aid of a computer program, I confirmed that the answer is yes: a standard set of additive conservation laws rules out as many unobserved reactions as possible. However, my analysis also shows that there are infinitely many other sets of predictively equivalent conservation. Thus particle physics faces a problem of global underdetermination: how to select a theory from a set of alternatives that make exactly the same predictions. In the case of conservation laws, it is possible to resolve the underdetermination by connecting laws with ontology: the standard set of laws correspond to a grouping of elementary particles into disjoint categories (i.e., the baryon, muon, electron and tau families). A nominalist might expect that these ontological categories themselves would be underdetermined by the data, but an analysis of the logic of additive conservation laws shows that this is not so: Any set of laws that (1) explains the absence of as many unobserved processes as possible, and (2) employs disjoint ontological categories, must agree with the ontology of the standard set of laws. In this case, two prob- 
lems are easier than one, because seeking a theory that reconciles ontology and dynamics constrains the alternatives more than considering ontology or dynamics in isolation.

We have encountered a number of standard themes from the philosophy of science in the course of this study: a plentitude principle, global underdetermination, the problem of induction, laws, simplicity and natural kinds. All these issues arise in practice, and physicists deal with them, albeit often implicitly as part of their research work rather than explicitly formulating the challenges as philosophical issues. Sometimes the solution is to appeal to a general principle, such as a plenitude principle, sometimes to pursue the implications of contextspecific assumptions, such as the logic of additive conservation principles. It is by studying the interplay of general principles and specific assumptions that we can see how scientists resolve the underdetermination of theory by evidence.

\section{Acknowledgments}

This research was supported by grants from the Social Sciences and Humanities Research Council of Canada (SSHRC) and from the Natural Sciences and Engineering Research Council of Canada (NSERC). I am indebted to Matthew Strassler, Manuella Vincter and the late Robert Coleman for helpful discussion of the physics of conservation laws. Mark Drew collaborated on the computational search for the most parsimonious set of conserved quantities. Alexandre Korolev, Leo Chen and Greg Dostatni provided valuable assistance with the particle and reaction databases and with the programs that infer a maximally strict set of conservation laws.

\section{Appendix: Formal Definitions and Proofs}

We begin with the vector representation of reactions due to Valdés-Pérez [38]. Suppose the known particles are enumerated as $p_{1}, \ldots, p_{n}$. In a given reaction $r$, we may count the number of occurrences of a particle $p$ among the reagents, which we denote by in $(p, r)$. For example, if $r=\mu^{-} \rightarrow e^{-}+\nu_{\mu}+\bar{\nu}_{e}$, then $i n\left(\mu^{-}, r\right)=1$, and $i n(p, r)=0$. If $r=p+p \rightarrow p+p+\pi^{0}$, then $i n(p, r)=$ 2. Similarly, we write $\operatorname{out}(p, r)$ for the number of occurrences of particle $p$ among the products of $r$. For example, out $\left(e^{-}, \mu^{-} \rightarrow e^{-}+\nu_{\mu}+\bar{\nu}_{e}\right)=1$, and out $\left(p, p+p \rightarrow p+p+\pi^{0}\right)=2$. Finally, define the net occurrence of particle $p$ in reaction $r$ by $\operatorname{net}(p, r) \equiv i n(p, r)-$ out $(p, r)$. Now we may represent a reaction as a vector as follows: For each reaction $r$, let $\mathbf{r}$ be the $n$-vector such that $\mathbf{r}(i)=\operatorname{net}\left(p_{i}, r\right)$, where $n$ is the total number of elementary particles, and 


\begin{tabular}{|l|c|c|c|c|c|c|c|}
\cline { 1 - 8 } Particle & 1 & 2 & 3 & 4 & 5 & 6 & 7 \\
\cline { 1 - 9 } Process/Quantum Number & $p$ & $\pi^{0}$ & $\mu^{-}$ & $e^{+}$ & $e^{-}$ & $v_{\mu}$ & $\bar{\nu}_{e}$ \\
\cline { 1 - 8 }$\mu^{-} \rightarrow e^{-}+\nu_{\mu}+\bar{\nu}_{e}$ & 0 & 0 & 1 & 0 & -1 & -1 & -1 \\
\hline$p \rightarrow e^{+}+\pi^{0}$ & 1 & -1 & 0 & -1 & 0 & 0 & 0 \\
\hline$p+p \rightarrow p+p+\pi^{0}$ & 0 & -1 & 0 & 0 & 0 & 0 & 0 \\
\hline Baryon Number & 1 & 0 & 0 & 0 & 0 & 0 & 0 \\
\hline Electric Charge & 1 & 0 & -1 & 1 & -1 & 0 & 0 \\
\hline
\end{tabular}

Table 2

The Representation of Reactions and Quantum Numbers as $n$-Dimensional Vectors

$\mathbf{r}(i)$ is the $i$-th entry in the vector $\mathbf{r}$. For example, if we take the 22 particles as numbered in Table 1 , the 22 -dimensional vector representing the process $\mu^{-} \rightarrow$ $e^{-}+\nu_{\mu}+\bar{\nu}_{e}$ corresponds to the process $p_{15} \rightarrow p_{19}+p_{17}+p_{22}$, represented by the vector $(0,0,0,0,0,0,0,0,0,0,0,0,0,0,1,0,-1,0,-1,0,0,-1)$. The process $p+p \rightarrow p+p+\pi^{0}$ is represented by the vector $\mathbf{r}$ where $\mathbf{r}(i)=0$ if $i \neq 9$ and $\mathbf{r}(i)=-1$ if $i=15$. Note that a quantum number $q$ can be represented as an $n$-dimensional vector as well. Table 2 illustrates the encoding of both reactions and quantum numbers as $n$-dimensional vectors. The proofs below use boldface to denote vectors.

Proof of Proposition 4. I now give the proof of Proposition 4, which implies that the matter-antimatter distinction is uniquely determined by the current reaction data.

Proposition 4 Let $Q, Q^{\prime}$ be any two predictively equivalent combinations of laws, with associated quantities. If all the quantities in $Q$ respect the matter/antimatter division, then so do all the quantities in $Q^{\prime}$. It follows that any combination of additive conservation laws that is predictively equivalent to the conservation of electric charge, baryon number, tau number, electron number and muon number respects the matter/antimatter division.

The proof proceeds by reformulating the proposition as the following theorem in linear algebra.

Let $B$ be a basis for a linear subspace $V$ such that all quantities in $B$ respect the matter/antimatter distinction. Then all quantities in any other basis $B^{\prime}$ for $V$ also respect the matter/antimatter distinction.

I assume that the sets of quantities $Q$ and $Q^{\prime}$ do not contain irredundant quantities; see Section 3. Then the two sets are predictively equivalent if and only if they are bases for the same linear space, so Proposition 4 is implied by the linear algebra proposition stated above, whose proof follows. 
Proof. Let $\mathbf{q}$ be a quantum number in $B^{\prime}$. As $B$ is a basis for $V$, we can write $\mathbf{q}$ as a linear combination of quantum numbers in $B=\left\{\mathbf{q}_{1}, . ., \mathbf{q}_{k}\right\}$ :

$$
\mathbf{q}=\sum_{l=1}^{k} a_{l} \mathbf{q}_{l}
$$

for suitable scalars $a_{l}$. Now suppose that particle $p_{i}$ is the antiparticle of particle $p_{j}$. Then since $B$ respects the matter/antimattter distinction, we have $\mathbf{q}_{l}(i)=-\mathbf{q}_{l}(j)$ for $l=1, . ., k$. By Equation 1 , we have $\mathbf{q}(i)=\sum_{l=1}^{k} a_{l} \mathbf{q}_{l}(i)$, which implies that

$$
\mathbf{q}(i)=\sum_{l=1}^{k} a_{l}\left(-\mathbf{q}_{l}(j)\right)=-\sum_{l=1}^{k} a_{l} \mathbf{q}_{l}(j) .
$$

Also from Equation 1 we have $\mathbf{q}(j)=\sum_{l=1}^{k} a_{l} \mathbf{q}_{l}(j)$, which by Equation 2 implies that $\mathbf{q}(i)=-\mathbf{q}(j)$. As this holds for every quantity $\mathbf{q} \in B^{\prime}$ and for every particle-antiparticle pair $p_{i}$ and $p_{j}$, it follows that $B^{\prime}$ respects the matter/antimatter distinction.

Proof of Theorem 5. Next, I prove Theorem 5, which establishes that predictively equivalent family law sets define the same particle families.

Theorem 5 Let $Q, Q^{\prime}$ be any two predictively equivalent combinations of laws, with associated quantities. If both $Q$ and $Q^{\prime}$ are family sets, then they define the same particle families as carriers of their quantities. It follows that if $Q^{\prime}$ is any family set of laws that is predictively equivalent to the conservation of baryon number, tau number, electron number and muon number, then $Q^{\prime}$ defines the same particle families as these laws (namely the baryons and the three lepton generations tau, electron and muon).

Theorem 5 follows from the following linear algebra theorem that asserts that any two family bases have the same carriers. Recall that carriers $(\mathbf{v})=$ $\{i \mid \mathbf{v}(i) \neq 0\}$, and say that $B$ is a family basis if for $\mathbf{v}_{1} \neq \mathbf{v}_{2}$ in $B$ we have carriers $\left(\mathbf{v}_{1}\right) \cap$ carriers $\left(\mathbf{v}_{2}\right)=\emptyset$. Say that a basis $B^{\prime}$ is a multiple of a basis $B$ if for every vector $\mathbf{v}^{\prime}$ in $B^{\prime}$, there is a vector $\mathbf{v}$ in $B$ and a scalar $a$ such that $\mathbf{v}^{\prime}=a \mathbf{v}$. If $\mathbf{v}=a \mathbf{v}^{\prime}$ for $a \neq 0$, then $\mathbf{v}$ and $\mathbf{v}^{\prime}$ have the same carriers, so the bases $B$ and $B^{\prime}$ determine the same families if $B^{\prime}$ is a multiple of a family basis $B$.

Suppose that $B, B^{\prime}$ are family bases for a linear space $V$. Then $B^{\prime}$ is a multiple of $B$. 
I assume that the sets of quantities $Q$ and $Q^{\prime}$ do not contain irredundant quantities; see Section 3. Then the two sets are predictively equivalent if and only if they are bases for the same linear space, so Theorem 5 is implied by the linear algebra proposition stated above, whose proof follows.

Proof. Let $\mathbf{v}^{\prime}$ be a vector in $B^{\prime}$. Then we may write $\mathbf{v}^{\prime}=\sum_{i=1}^{n} a_{i} \mathbf{v}_{i}$, where $\mathbf{v}_{i} \in B$, with $n=\operatorname{dim}(B)$. Since $B^{\prime}$ is a basis, $\mathbf{v}^{\prime} \neq \mathbf{0}$ and there exists $a_{i} \neq 0$. Thus

$$
\operatorname{carriers}\left(\mathbf{v}_{i}\right) \subseteq \operatorname{carriers}\left(\mathbf{v}^{\prime}\right)
$$

because $B$ is a family set. We may write

$$
\mathbf{v}_{i}=\sum_{k=1}^{n} b_{k} \mathbf{v}_{k}^{\prime}+b \mathbf{v}^{\prime},
$$

where $\mathbf{v}_{k}^{\prime} \neq \mathbf{v}^{\prime}$ is in $B^{\prime}$. Since $B^{\prime}$ is a family set, the carriers of $\sum_{k=1}^{n} b_{k} \mathbf{v}_{k}^{\prime}$ are disjoint from those of $\mathbf{v}^{\prime}$. As the carriers of $\mathbf{v}^{\prime}$ include those of $\mathbf{v}_{i}$, it follows that $\mathbf{v}_{i}=b \mathbf{v}^{\prime}$ where $b \neq 0$, and so $\mathbf{v}^{\prime}=\frac{1}{b} \mathbf{v}_{i}$. As this holds for any vector in $B^{\prime}$, it follows that $B^{\prime}$ is a multiple of $B$.

Proof of Theorem 6. I prove the main result: taking a logically independent quantity (e.g., electric charge) as given, particle families are uniquely determined by the data.

Theorem 6 Let $Q$ be a family set of laws, with at least two associated quantities $\left\{q_{1}, . ., q_{m}\right\}$, and consider a quantity $q$ that is logically independent of the quantities $\left\{q_{1}, . ., q_{m}\right\}$ associated with $Q$. Let $Q^{\prime}$ be any other family set of laws, with associated quantities $\left\{q_{1}^{\prime}, \ldots, q_{m}^{\prime}\right\}$, such that the conservation of all quantities $\left\{q, q_{1}^{\prime}, \ldots, q_{m}^{\prime}\right\}$ permits exactly the same reactions as the conservation of all quantities $\left\{q, q_{1}, \ldots, q_{m}\right\}$. Then $Q$ and $Q^{\prime}$ define the same particle families as carriers of their quantities. It follows that if $Q^{\prime}$ is any family set of laws that together with electric charge is predictively equivalent to the conservation of electric charge, baryon number, tau number, electron number and muon number, then $Q^{\prime}$ defines the same particle families as baryon number, tau number, electron number and muon number (namely the baryons and the three lepton generations tau, electron and muon).

Theorem 6 follows from the following linear algebra theorem.

Theorem 7 Let $B \cup\{\mathbf{q}\}$ be a basis for $V$ such that $|B|>2, B$ is a family set and $\mathbf{q}$ is logically independent of $B$. Let $B^{\prime}$ be another family set such that $B^{\prime} \cup\{\mathbf{q}\}$ is a basis for $V$. Then $B^{\prime}$ is a linear multiple of $B$.

Theorem 6 follows if we set $Q:=B$ and $Q^{\prime}:=B^{\prime}$.

Proof. Let $\mathbf{v}_{q}$ be the vector $\mathbf{v}$ with 0 assigned to all carriers of $\mathbf{q}$ (i.e. $\mathbf{v}_{q}(i)=0$ if $\mathbf{q}(i) \neq 0$, and $\mathbf{v}_{q}(i)=\mathbf{v}(i)$ otherwise). For a set of vectors $U$, let $U_{q}=\left\{\mathbf{v}_{q}\right.$ : 
$\mathbf{v} \in U\}$. It is easy to verify that $B_{q}$ and $B_{q}^{\prime}$ are family bases for $V_{q}$, so the previous Proposition implies that $B_{q}^{\prime}$ is a multiple of $B_{q}$. Thus every quantity $\mathbf{v}_{i}^{\prime}$ in $B^{\prime}$ is of the form

$$
\mathbf{v}_{i}^{\prime}=a_{i} \mathbf{v}_{i}+b_{i} \mathbf{q}
$$

for some vector $\mathbf{v}_{i} \in B$; we argue by contradiction that $b_{i}=0$ for all $\mathbf{v}_{i}^{\prime} \in B^{\prime}$, which establishes the theorem.

Case 1: there are two distinct $\mathbf{v}_{i}, \mathbf{v}_{j}$ such that $b_{i} \neq 0$ and $b_{j} \neq 0$. Since $|B|>2$, there is a $\mathbf{v}_{k}$ different from $\mathbf{v}_{i}, \mathbf{v}_{j}$. As $B$ is a family set and $\mathbf{q}$ is logically independent of $B$, this implies that there is a particle $p$ carrying both $\mathbf{v}_{k}$ and $\mathbf{q}$ but neither $\mathbf{v}_{i}$ nor $\mathbf{v}_{j}$. So $p$ carries $\mathbf{v}_{i}^{\prime}=a_{i} \mathbf{v}_{i}+b_{i} \mathbf{q}$ and also $\mathbf{v}_{j}^{\prime}=a_{j} \mathbf{v}_{j}+b_{j} \mathbf{q}$, which contradicts the supposition that $B^{\prime}$ is a family set.

Case 2: there is exactly one $\mathbf{v}_{i}^{\prime}$ such that $b_{i} \neq 0$. Choose a vector $\mathbf{v}_{j}$ and a particle $p$ such that $p$ carries both $\mathbf{v}_{j}$ and $\mathbf{q}$, but not $\mathbf{v}_{i}$. Then $p$ carries $\mathbf{v}_{i}^{\prime}=a_{i} \mathbf{v}_{i}+b_{i} \mathbf{q}$, and since $\mathbf{v}_{j}^{\prime}=a_{j} \mathbf{v}_{j}$, the particle $p$ carries $\mathbf{v}_{j}^{\prime}$ as well, which contradicts the supposition that $B^{\prime}$ is a family set.

In either case we arrive at a contradiction, which shows that $B^{\prime}$ is a linear multiple of $B$.

\section{References}

[1] O-M. Bilaniuk and E.C. George Sudarshan. Particles beyond the light barrier. Physics Today, 22:43-52, 1969.

[2] Leon Cooper. Physics: Structure and Meaning. University Press of New England, Hanover, 1970.

[3] W.N. Cottingham and D.A. Greenwood. An introduction to the standard model of particle physics. Cambridge : Cambridge University Press, 2nd edition, 2007.

[4] Chris Daly. Natural kinds. In Routledge Encyclopaedia of Philosophy. Routledge, London, 1998.

[5] John Earman. Laws, symmetry, and symmetry breaking: Invariance, conservation principles, and objectivity. Philosophy of Science, 71:1227-1241, December 2004.

[6] S. Eidelman and The Particle Data Group. The Review of Particle Physics. Phys. Lett. B, 592(1), 2004.

[7] Richard Feynman. The Character of Physical Law. MIT Press, Cambridge, Mass., 19; 1990 edition, 1965.

[8] K.W. Ford. The World of Elementary Particles. Blaisdell, New York, 1963. 
[9] Kazuo Fujikawa and Hiroshi Suzuki. Path Integral and Quantum Anomalies. Number 122 in International Series on Monographs on Physics (ISMP). Oxford Science Publications, 2004.

[10] Murray Gell-Mann and Yuval Ne'eman. The eightfold way. W.A. Benjamin, New York, 1964.

[11] N. Goodman. Fact, Fiction and Forecast. Harvard University Press, Cambridge, MA, 1983.

[12] Durham Database Group. Hepbase. URL = http://durpdg.dur.ac.uk/hepdata/reac.html, 2007.

[13] S. Jain, D. Osherson, J. S. Royer, and A. Sharma. Systems That Learn. M.I.T. Press, 2 edition, 1999.

[14] R. Kane. Principles of reason. Erkenntnis, 24:115-36, 1986.

[15] Ravindran Kannan and Achim Bachem. Polynomial algorithms for computing the smith and hermite normal forms of an integer matrix. SIAM Journal of Computing, 8(4):499-507, 1979.

[16] Kevin T. Kelly. The Logic of Reliable Inquiry. Oxford University Press, Oxford, 1996.

[17] Hilary Kornblith. Inductive Inference and Its Natural Ground. MIT Press, Cambridge, Mass., 1993.

[18] Thomas Kuhn. The Structure of Scientific Revolutions. University of Chicago Press, Chicago, 1970.

[19] David Lewis. Counterfactuals. Harvard University Press, Cambridge, Mass., 1973.

[20] David Lewis. New work for a theory of universals. Australasian Journal of Philosophy, 61:4:343-377, 1983.

[21] David J. C. MacKay. Information Theory, Inference and Learning Algorithms. Cambridge University Press, Cambridge, U.K., 2003.

[22] Yuval Ne'eman and Yoram Kirsh. The Particle Hunters. Cambridge University Press, Cambridge, 1983.

[23] Roland Omnes. Introduction to Particle Physics. Wiley Interscience, London, New York, 1971.

[24] Hilary Putnam. 'degree of confirmation' and inductive logic. In A. Schilpp, editor, The Philosophy of Rudolf Carnap. Open Court, La Salle, Ill., 1963.

[25] Hilary Putnam. Trial and error predicates and a solution to a problem of mostowksi. Journal of Symbolic Logic, 30:49-57, 1965.

[26] Hilary Putnam. Probability and confirmation. In Mathematics, Matter and Method, Philosophical Papers, volume 1. Cambridge University Press, London, 1975. 
[27] W. V. Quine. Natural kinds. In Ontological Relativity and Other Essays, pages 114-138. Columbia University Press, New York and London, 1969.

[28] H. Reichenbach. The Theory of Probability. Cambridge University Press, London, 1949.

[29] Wesley Salmon. Hans reichenbach's vindication of induction. Erkenntnis, 35:99$122,1991$.

[30] Oliver Schulte. Means-ends epistemology. The British Journal for the Philosophy of Science, 79(1):141-147, 1996.

[31] Oliver Schulte. Inferring conservation laws in particle physics: A case study in the problem of induction. The British Journal for the Philosophy of Science, 51:771-806, 2000.

[32] Oliver Schulte. Data sets and programs for particle reaction analysis. URL $=$ http://www.cs.sfu.ca/ oschulte/particles/, 2007.

[33] Oliver Schulte. Formal learning theory. In Edward N. Zalta, editor, The Stanford Encyclopedia of Philosophy. Spring 2006.

[34] Lawrence Sklar. Methodological conservatism. Philosophical Review, LXXXIV:374-400, 1975.

[35] Paul Tipler and Ralph Llewellyn. Modern Physics. W. H. Freeman, 4 edition, 2002.

[36] Raúl Valdés-Pérez. On the justification of multiple selection rules of conservation in particle physics phenomenology. Computer Physics Communications, 94:25-30, 1996.

[37] Raúl Valdés-Pérez and Michael Erdmann. Systematic induction and parsimony of phenomenological conservation laws. Computer Physics Communications, 83:171-180, 1994.

[38] R.E. Valdés-Pérez. Algebraic reasoning about reactions: Discovery of conserved properties in particle physics. Machine Learning, 17:47-67, 1994.

[39] Bas van Fraassen. The Scientific Image. Clarendon Press, Oxford, 1980.

[40] S. Weinberg. The Quantum Theory of Fields. Volume II: Modern Applications. Cambridge University Press, 2001.

[41] William S.C. Williams. Nuclear and Particle Phyics. Oxford University Press, New York, 1997.

[42] L. Wolfenstein and T.G. Trippe. Tests of conservation laws. In The Review of Particle Physics [6]. 